IdeAs

Idées d'Amériques

$4 \mid 2013$

Crises et effets de crise dans les Amériques

\title{
Les solutions de sortie de crise sont en crise
}

Jean-Michel Saussois

\section{OpenEdition}

Journals

Édition électronique

URL : https://journals.openedition.org/ideas/649

DOI : 10.4000/ideas.649

ISSN : 1950-5701

Éditeur

Institut des Amériques

Référence électronique

Jean-Michel Saussois, "Les solutions de sortie de crise sont en crise », IdeAs [En ligne], 4 | 2013, mis en ligne le 25 octobre 2013, consulté le 18 octobre 2022. URL : http://journals.openedition.org/ideas/ 649 ; DOI : https://doi.org/10.4000/ideas.649

Ce document a été généré automatiquement le 18 octobre 2022

\section{(c) (1) (9)}

Creative Commons - Attribution - Pas d'Utilisation Commerciale - Pas de Modification 4.0 International - CC BY-NC-ND 4.0

https://creativecommons.org/licenses/by-nc-nd/4.0/ 


\title{
Les solutions de sortie de crise sont en crise
}

\author{
Jean-Michel Saussois
}

\section{Introduction}

1 Une démarche à suivre pour penser la sortie de crise, la pire que le capitalisme eût à connaitre depuis celle de 1929, pourrait être celle de la lucidité. La lucidité décrite par l'écrivain allemand Walter Benjamin (2003) lorsqu'il décrivait le travail de Berthold Brecht. En pleine période nazie, Brecht avait inventé un théâtre en rupture complète avec le théâtre qui entretenait l'illusion - une illusion que les spectateurs viennent précisément rechercher au théâtre mais aussi dans la politique qui théâtralise l'illusion, non sans efficacité d'ailleurs. Les hommes providentiels savent fort bien jouer de cette théâtralisation. Brecht dit au public que l'homme peut vivre dans le non espoir à partir du moment où il sait comment il en est arrivé là. Brecht nous explique, par exemple, dans l'une de ses grandes pièces, l'opéra de quat'sous, pourquoi la prophétie de Jenny la serveuse, ne fût pas réalisée ${ }^{1}$. Vivant, Brecht nous aurait expliqué pourquoi Cuba est devenu selon le mot meurtrier de la romancière cubaine, Zoé Valdès, un «socialismland». Il nous aurait aussi expliqué ce que signifie un système de domination économique lorsque des hommes osent publiquement l'affronter. Lumumba, Allende, Mossadegh, la liste serait longue. Pour expliquer la crise financière, Brecht vivant aurait certainement mis en scène l'irrésistible ascension de Lehmann Brother ou de Goldman Sachs.

2 A la question dérangeante du type « comment en est-on arrivé là ? », la pédagogie doit guider la réponse. La crise financière de 2008 a le mérite de faire surgir l'exaspération comme caractérisant le capitalisme actuel. C'est un cas pédagogique, un cas d'école où toutes les composantes du capitalisme sont poussées à leur limite, jusqu'à l'absurde. L'enchevêtrement des acteurs du système est un peu comme à l'image du jeu du mikado : il suffit d'une baguette déplacée un peu brutalement pour que l'empilement des baguettes s'écroule tout d'un coup. Ici la baguette déplacée brutalement a été la 
banque d'affaires Lehmann Brother, banque non renflouée par l'État fédéral américain qui souhaitait en faire un exemple. Cette faillite spectaculaire a fait basculer dans la crise le secteur bancaire américain et cela dans un temps court, non seulement sur le continent américain mais aussi sur le continent européen. Juste rappel du fait que le capitalisme est à comprendre comme un système sous la forme d'une hiérarchie enchevêtrée entre les grandes entreprises, les banques, les assurances, les fonds de pensions, les hedge funds, les cabinets d'audit, les courtiers, les agences de notation. Comment est-on arrivé à cet empilement fragile d'intérêts aussi complexe et à cette bascule aussi rapide?

3 Ce résultat est le fait d'une inventivité spectaculaire des opérateurs sur les marchés financiers qui n'ont eu de cesse de trouver des produits bancaires qui échappent à la prudence des banques de dépôt lorsqu'elles prêtent de l'argent aux entreprises ou aux particuliers. Cette inventivité a été rendue possible par la déréglementation bancaire aux Etats-Unis - et cela dès les années 70 - mais aussi par l'introduction de nouvelles règles comptables qui valorisent les actifs non pas à leur valeur historique mais au prix du marché du jour. Plus les actifs prennent de la valeur et plus le niveau d'endettement est faible rapporté à la valeur des actifs engagés sur un prêt. Plus les banques s'endettent et plus elles peuvent prêter! L'argent se nourrit de l'argent, une façon de caractériser l'exaspération du capitalisme aujourd'hui. Ce que l'on a appelé la financiarisation de l'économie revient à dire que le crédit devient, en fait, le levier de la croissance des économies qui s'éloignent ainsi de la croissance par l'innovation. Les montants des crédits, dont les crédits immobiliers, explosent à des niveaux jamais atteints sans rapport avec l'activité, d'où la formation de bulles.

Les ingénieurs recrutés à grands frais par les banques s'en donnent alors à cœur joie pour trouver des outils dont la vertu est de diluer les risques au maximum. Les banques vendent leur risque de non paiement de leurs emprunteurs en achetant des protections à des spécialistes de l'évaluation des risques. Sans entrer dans le détail des techniques sophistiquées de finance de marché, il suffit de comprendre que ces outils ont pour seul but de transférer les risques d'une banque à une autre, de façon à ne pas avoir à mettre en face d'un crédit un capital, au cas où les choses viendraient à mal tourner. Le crédit accordé devient le mistigri que les banques se repassent entre elles moyennant commissions croisées, le but du jeu étant évidemment de ne pas faire apparaitre ces transactions au bilan des banques. Tout se joue hors bilan, une affaire de périmètre en quelque sorte.

5 Cet enchevêtrement est d'autant moins visible que la valeur des actifs - dont les actifs immobiliers - continue d'augmenter et rend, du même coup, le prêteur serein dans la mesure où il pourra toujours se faire rembourser en vendant des actifs qui ont pris de la valeur.

6 Cet empilement spectaculaire des crédits a été rendu possible par de nouveaux venus spécialistes en statistiques et mathématiques qui cherchent à minimiser les risques en jouant sur des effets d'échelle. L'idée simple est de réunir dans un grand réservoir tous les titres qui sont en fait des contreparties de crédit dont on ne connaît plus l'origine. Le risque ne porte plus alors sur madame $\mathrm{X}$ mais plutôt sur des milliers de titres qui renvoient eux-mêmes à des crédits de différentes natures, allant de l'immobilier pour les pauvres aux cartes de crédit, à la consommation ou aux crédits des étudiants pour payer leurs études. Les banques font appel à des ingénieurs mathématiciens qui ont alors l'idée de structurer ces milliers de titres en plusieurs familles selon la nature du 
risque encouru. On assiste alors à la fabrication d'un véritable mille feuille. Pour les acheteurs de titres qui sont prudents et qui ont peur du risque, on fabriquera une couche spéciale appelée senior ou super senior! Faut-il parler de cynisme ou de complot fomenté par ceux qui savent vis-à-vis de ceux qui ne savent pas?

7 Comme aimait le souligner le grand historien du capitalisme Fernand Braudel, «le capitalisme n'est ni bon, ni mauvais, ni moral, ni tricheur; il est comme il est et le problème n'est pas de le juger, mais de le comprendre $\|^{2}$. Comprendre pour ensuite en tirer des leçons, voilà la ligne. Voilà, en quelques paragraphes, le parti pris brechtien qui n'est finalement qu'une reprise $\mathrm{du}$ bon vieux dicton de la philosophie scolastique: " non indignari nec flere sed intellegire» ou "ne pas s'indigner, ni pleurer mais comprendre ». Cette démarche un peu austère contient en elle même un effet de douche "glaçante » pour ceux qui ont la charge de proposer des solutions. Aujourd'hui, les hommes politiques hésitent à manier une pédagogie lucide, rechignent souvent à appeler un chat un chat, une crise conjoncturelle une crise structurelle. Mieux vaut faire rêver. Il faut reconnaître que le rêve aide mieux à vivre que la lucidité. La lucidité assèche la vie quotidienne. Hegel disait d'ailleurs que l'illusion n'était pas illusoire. Godot l'européen attendra alors la croissance.

Un dernier mot sur cet appel à la lucidité pédagogique pour penser la sortie de crise. Comprendre pour agir est le contraire de s'en remettre au destin, le fatum des Romains. Je reprends volontiers à mon compte la remarque de Schumpeter accusé de défaitisme dans sa réponse à la question qu'il se posait à lui-même: le capitalisme peut-il survivre? «Le compte rendu qu'un navire est en train de couler n'est pas défaitiste. Seul peut l'être l'esprit dans lequel il est pris connaissance de ce compterendu : l'équipage peut se croiser les bras et se noyer. Mais il peut aussi courir aux pompes (Schumpeter, 1990)».

9 Les hommes politiques courent aujourd'hui aux pompes avec plus ou moins d'entrain pour écoper. L'intention ici est donc de montrer que les solutions de crise sont elles mêmes en crise, ce qui ne rend pas la tâche facile

\section{Les solutions de crise sont en crise}

10 Tout diagnostic porte en lui-même sa solution, d'où la nécessité de porter le bon diagnostic au bon moment. Une douleur diagnostiquée comme cardiaque appelle la solution du cardiologue plutôt que celle du podologue. Les nombreux diagnostics portés sur la crise appellent alors des solutions de nature et d'ampleur différentes.

\section{La solution cosmétique}

11 Pour les économistes qui se réclament du paradigme de l'efficience, le diagnostic est simple : ce ne sont pas les mécanismes financiers qui sont intrinsèquement fautifs mais le fait que les titres aient été mal conçus en amont par des spécialistes des mathématiques financières et d'évaluation statistique du risque. Une telle analyse ne remet pas en cause l'idée que les marchés permettent une allocation efficiente du capital. La logique de l'interconnexion universelle permettant une circulation totalement libre du capital, sous toutes ses formes, n'y est pas remise en question. La crise est avant tout une affaire d'opacité. Aux États-Unis plus qu'en Europe ou en Amérique latine, dans la mesure où c'est dans ce pays que l'innovation financière a été 
la plus vivace, la titrisation des crédits immobiliers a échoué parce qu'elle a produit des obligations qui peuvent se retrouver être cotées, et dont personne n'est plus capable d'évaluer avec précision les risques. La chaine de montage des crédits est tellement longue et complexe que plus personne ne sait quel est le véritable détenteur de la créance. Si le diagnostic porte sur l'opacité, la solution porte alors sur la transparence. Si la transparence ne convainc pas les acteurs ou si les acteurs font semblant et ne jouent pas le jeu, alors la transparence doit être imposée aux acteurs qui traînent les pieds. Les citoyens ont le droit de savoir. Des solutions beaucoup plus fortes sur le plan symbolique peuvent aussi être proposées et l'on sait l'importance du symbolique en politique. Ces solutions visent à rendre transparents les montants de primes des traders et les salaires des dirigeants voire les patrimoines des élus. Le citoyen a finalement le droit de savoir et c'est en sachant que son jugement s'affinera.

\section{La solution morale}

Le diagnostic de la crise porte plutôt sur une crise de foi. Des milliers de salariés qui ne doutaient pas dans les années 60 des bienfaits du capitalisme se mettent à douter ; en fait, les cyniques ont chassé les croyants en se comportant ouvertement en prédateurs, découpant sans vergogne des grandes entreprises qui avaient pignon sur rue et qui étaient appréciées des pères de famille à la recherche de dividendes modestes mais stables sur une longue durée. On se réfère ici à un capitalisme managérial, dans la main des dirigeants et non des actionnaires. La solution consiste alors à retrouver les valeurs fondamentales du capitalisme des grandes entreprises pour rétablir la confiance perdue aussi bien du côté des salariés qui savent qu'ils ne feront plus carrière dans une seule entreprise que du côté des petits actionnaires qui se sentent dupés par les gestionnaires de fonds de pension. Les opérateurs sont allés trop loin dans leur cupidité et il faut retrouver l'esprit initial du capitalisme et punir les traîtres, ceux qui ont franchi les limites de la décence. Il faut alors extraire les pommes pourries du tonneau et le faire savoir à l'opinion publique qui sera ainsi rassurée de voir les coupables punis. Dans un livre passionnant sur Enron, Anne Frison-Roche (2003) montre la vitesse avec laquelle les acteurs du système, politique et économique, ont réagi par souci de survie. Le Sénat américain a vite mis sur pied une commission d'enquête et a crée un conseil doté de moyens importants pour contrôler la profession d'auditeur. Les autorités politiques font savoir aux actionnaires que les craquements ont été écoutés et compris. Les brebis galeuses sont écartées à la vue de tous. Forme moderne du pilori installé sur la place de grève, l'un des hauts responsables d'Enron est arrêté en pleine rue de New York et apparaît à la télévision menottes aux poings. Le capitalisme américain fait amende honorable, jurant que, cette fois ci, on ne le reprendrait plus à tricher avec les règles édictées et non suivies par des francs-tireurs. Qui est « on »? Voilà une question dont les réponses supposent une décomposition plus fine du système. La pomme rapidement désignée comme pourrie (rotten apple) sera extraite du tonneau sans faire de relation entre la pomme et le tonneau, c'est-à-dire le mécanisme de pourrissement. Qu'a-t-on appris de cette crise, pourtant prémonitoire de la faillite à venir du capitalisme financier aux États-Unis? Peu de choses finalement, la poussière ayant été trop rapidement remise sous le tapis. Enron semble d'ailleurs relever aujourd'hui de l'histoire immédiate, une faillite somme toute relative compte tenu de l'ampleur des faillites en chaîne dans le secteur des banques et des assurances américaines. Si l'on isole un seul facteur, le facteur moral, pour expliquer la crise, il y a de fortes chances de 
ne rien apprendre de cette crise dans la mesure où l'on se trouve en face de niveaux enchevêtrés, sur lesquels agissent des forces organisationnelles qui, elles, sont portées par des acteurs qui défendent des intérêts, explicites ou latents, peu importe. Ces acteurs peuvent être des configurations d'organisations, mais aussi des acteurs en chair et en os. Ce travail sur la compréhension des hiérarchies (privées et publiques) enchevêtrées reste à faire.

\section{La solution par la régulation}

13 Ce diagnostic ignore le diagnostic précédent car il est vain de vouloir moraliser le capitalisme, vœu pieux sympathique mais qui reste un vœu pieux. Le capitalisme est par essence amoral. En suivant ce diagnostic, la crise est avant tout un excès du mouvement de dérégulation des années 70 . La solution passe par une re-régulation notamment des marchés financiers. Cette solution, plutôt à dominante juridique, passe par une refonte complète des lois et réglementations qui encadrent le capitalisme selon l'agenda suivi par Roosevelt dès 1933 pour reconstruire le marché financier après la crise de 29. Si on suit ce diagnostic à dominante juridico-administratif, s'ouvre alors une solution qui se donne pour objectif de combattre l'emprise excessive de la liquidité sur le financement et les décisions d'investissement. La solution passe alors par le cloisonnement des circuits et des acteurs de façon à limiter les contagions, à la manière du Glass Steagal Act de 1933 qui interdit aux banques de dépôt d'intervenir sur les marchés financiers de façon à les préserver des emballements boursiers.

\section{La solution politique}

14 Le diagnostic est plus politique qu'économique. Ce sont des politiques qui commencent à douter d'une solution par la relance via les dépenses publiques, même s'ils restent hantés par le chômage ( 25 \% de la population active américaine en 1934 et cela quatre ans après la grande crise).

Les politiques ne croient plus trop (même s'ils n'expriment pas publiquement leur doute) aux solutions qui passent par la régulation car ils savent bien que les régulés finissent toujours par contrôler les régulateurs. Les agences de régulation ne sont pas sans mérite car elles permettent de re-légitimer le capitalisme mais elles ne font pas toutes la preuve de leur efficacité, le meilleur exemple étant le mutisme de la Security Exchange Commission pendant la crise de 2008. Bien que nécessaires, ces agences de régulation ne sont pas suffisantes.

16 Leur diagnostic est plus profond. La contradiction porte sur le fait que le capitalisme managérial et financier ignore les frontières, aime la fluidité : fluidité de capitaux, fluidité d'hommes, fluidité des idées. Le capitalisme n'aime pas être fixé sur un territoire ou sur un sol. Les États, eux, exercent leur souveraineté à l'intérieur de frontières et assurent la protection de leurs citoyens qui travaillent sur leur territoire et qui sont aussi des contribuables. Les grandes entreprises ignorent ce type d'analyse et ont une vision mondiale et non nationale ni internationale. Les stratégies d'optimisation fiscale des grandes entreprises illustrent ce comportement. La solution passe alors par la main visible des politiques et non par celle des dirigeants. Elle appelle des hommes politiques au profil churchillien qui se persuadent qu'il ne faut pas abandonner la partie et qu'elle reste jouable même si c'est difficile. Toujours prêt à 
renaître sous des formes nouvelles, le capitalisme ne répond pas à des lois immuables (Saussois, 2011). Les mesures protectionnistes au niveau d'un État ou de plusieurs États (Communauté européenne) sont alors évoquées comme seules capables de rétablir les salaires réels à un niveau acceptable, en dépit des risques qu'elles contiennent vis-à-vis des pays exportateurs. Il en va de même pour retrouver la souveraineté fiscale.

\section{La solution révolutionnaire}

Pour ceux qui militent pour la destruction du capitalisme par une alternative apocalyptique du type socialisme ou barbarie, les crises sont positives et la sociale démocratie devient un obstacle à la solution révolutionnaire dans sa volonté de réformer le capitalisme. Ces militants sont de moins en moins nombreux en France mais aussi dans le monde. Les Tupamaros ont rejoint aujourd'hui les rangs de la jeune démocratie politique en Uruguay. Lula Da Silva abandonna lui aussi sa position de militant syndicaliste pour diriger l'un des plus grands pays d'Amérique Latine. Voilà un dirigeant politique qui souhaitait être le chef de file des pays émergents pour peser sur une transformation radicale des équilibres politiques au sein des organisations internationales comme l'ONU, le FMI ou l'OMC. Son plaidoyer (Lula da Silva, 2005) pour trouver des champs communs entre Davos et Porto Allègre transcendait les oppositions des pays riches et des pays pauvres, et cela, au nom de l'humanité : « Il ne s'agit pas de demander aux gens de cesser d'être ce qu'ils sont, mais bien d'établir des liens entre des communautés unies par une destinée humaine indivisible ».

\section{Conclusion}

Si les solutions de crise sont elles mêmes en crise, dans un univers qui se recompose selon des lignes de force différente, il nous faut trouver un nouveau cadre conceptuel qui permette de saisir la trame qui définit les sociétés dans lesquelles nous vivons aujourd'hui. Les historiens ont fait ce travail consistant à débusquer ce qu'ils appellent les facteurs saillants. Ils nous expliquent, par exemple, qu'il faut comprendre la Grèce comme une civilisation de la mer d'Egée de la Trace à la Crète, puis qu'il faut comprendre l'Egypte comme une civilisation du Nil domestiqué. Reste à trouver l'équivalent de la mer Méditerranée ou du Nil pour comprendre ce qui nous arrive aujourd'hui. Je propose comme facteur saillant le fait que les grandes entreprises (Saussois, 2012 : 531-557) sont en train de devenir l'acteur dominant d'une économie mondialisée et que ces organisations économiques deviennent des institutions politiques, mais des institutions qui connaissent elles aussi une crise, une crise de légitimité auprès de la société civile.

19 L'historien Alfred Chandler dans son magistral travail de fresque utilise bien l'expression managerial capitalism pour qualifier la dimension organisationnelle de l'économie mais sans en tirer les conséquences politiques en termes de pouvoir et de contrôle. Les grandes entreprises produisent de l'efficacité dans des secteurs bien spécifiques comme les secteurs agro-alimentaires ou chimiques ou pharmaceutiques ou informatiques où s'articulent, grâce à l'action de milliers de managers, la production de masse et la distribution de masse, tout cela pour assurer une croissance durable. Certes, cette distinction est nécessaire mais pas suffisante pour comprendre ce phénomène des grandes entreprises qui s'emparent, sous nos yeux, des espaces publics et qui se 
détachent volontairement des lois du marché tout en concurrençant, par leur puissance économique, les politiques publiques des États voire des organisations supranationales comme l'Union européenne ou l'Alena. Ces grandes entreprises ne produisent pas de l'efficacité dans un espace vide, elles se développent et se transforment aussi en produisant des arrangements politiques et sociaux. Autre point important, ces grandes entreprises diffusent en permanence des valeurs, elles ne cessent de construire et de reconstruire leur légitimité auprès d'une opinion publique dont il faut sans cesse gagner les faveurs.

Les grandes entreprises publiques et privées non seulement fabriquent continuellement des images publiques mais constituent en elles mêmes des institutions avec leur mode de gouvernement privé et de reproduction des élites. Qu'elles soient publiques ou privées ne change rien à l'affaire. Les dirigeants des grands groupes « parlent» de plus en plus comme des responsables politiques et proposent même de constitutionnaliser l'entreprise en intégrant les parties prenantes (Frérot, 2013) ${ }^{3}$. L'économique double en permanence le politique. Ces grandes entreprises plongent toujours leurs racines dans le marché mais s'en affranchissent fortement. Sans pouvoir nommer ce phénomène autrement que par capitalisme des organisations, voilà ce qui me semble caractériser le capitalisme aujourd'hui. C'est cette piste de recherche qu'il me semble urgent d'explorer avec sérénité, sans slogans usés jusqu'à la corde comme les attaques ad hominem contre les grands monopoles ou le big business qui incarnerait le mal par rapport au small business qui serait paré de toutes les vertus, avec le fameux slogan small is beautiful. Ces grandes entreprises sont en train de donner le tempo d'une musique souvent coproduite avec des États qui se trouvent eux-mêmes en difficulté mais qui gardent le monopole de la violence légitime si la résignation laisse la place à la révolte...

\section{BIBLIOGRAPHIE}

Benjamin, Walter, Écrits français, Gallimard, Paris, 2003.

Braudel, Fernand, La dynamique du capitalisme, Paris, Flammarion, 2008.

Brecht, Berthold, Théâtre complet tome 2 (1928-1931), L'Arche, 1988.

Frérot, Antoine, « Inviter toutes les parties prenantes à la gouvernance », Le Monde, 13 juin 2013.

Frison-Roche, Marie-Anne, Les leçons d'Enron - Capitalisme, la déchirure, Autrement, Paris, 2003.

Lula da Silva, Luis Inacio, «Entre Davos et Porto Alegre, des champs communs possibles », Le Monde, 27 janvier 2005.

Robé, Jean-Philippe, «L'entreprise et la constitutionnalisation du système-monde », in Roger Baudoin (dir.), L'entreprise, formes de la propriété et responsabilités sociales, Collège des Bernardins, Paris 2012, p. 273-344.

Saussois, Jean-Michel, Capitalisme : un dieu sans bible, Paris, Cavalier Bleu, 2011. 
Saussois, Jean-Michel, « La grande entreprise : un objet dont la sociologie reste à faire » in Roger Baudoin (dir.), L'entreprise, formes de la propriété et responsabilités sociales, Collège des Bernardins, Paris 2012, p. 531-557.

Schumpeter, Joseph, (1952), Capitalisme, socialisme et démocratie, traduction de G. Fain, Payot, 1990.

\section{NOTES}

1. Voir Brecht (1988).

2. Voir Braudel (2008).

3. Président directeur général de Veolia Environnement. Sur la question de la constitutionnalisation, on se reportera également au chapitre de Jean-Philippe Robé (2012).

\section{AUTEUR}

\section{JEAN-MICHEL SAUSSOIS}

Jean-Michel Saussois est professeur émérite à ESCP Europe, CERALE où il enseigne un cours sur l'« Organisation et management » et un second sur le management et l'évaluation des politiques publiques. Il est également directeur du Centre de Recherche et Développement en Management public. Depuis 1998, il est consultant à l'OCDE ; il a codirigé un projet sur la gestion des connaissances qui a été publié par l'OCDE en 2001 Knowledge management in the learning society. Jean Michel Saussois est, par ailleurs, membre des comités de rédaction de Sociologie du Travail, Politiques et Management Public. Les derniers ouvrages qu'il a publiés s'intitulent Le capitalisme sans répit (La Dispute, 2006) et Théories des organisations (collection Repères, La Découverte, 2007). saussois@escpeurope.eu 